\section{Complementary use of topical bitter melon for atopic dermatitis}

\author{
Dai Park, Nguyen P. Tran, \\ Jerald M. Duncan, D. Betty Lew \\ Children's Foundation Research Center \\ at Le Bonheur Children's Hospital and \\ University of Tennessee Health Science \\ Center, Memphis, Tennessee, USA
}

\section{Abstract}

Momordica charantia (bitter melon) is popular in systems of traditional medicine to treat a variety of diseases including atopic dermatitis, which is an inflammatory, chronically relapsing skin disorder characterized by dry, scaly, pruritic skin. While there is growing community interest in adopting bitter melon as a complementary medicine, there are no clinical studies looking at its use for atopic dermatitis. Here we report a case of a 6-yearold female with severe refractory atopic dermatitis that responded to treatment with topical bitter melon in an open half-side comparison trial.

\section{Introduction}

Atopic dermatitis is a highly prevalent, chronic, relapsing inflammatory skin disorder which occurs most frequently in children but affects adults as well. It is often associated with elevated IgE levels and a personal or family history of allergies, allergic rhinitis, and asthma. Its pathogenesis is complex and not completely understood. The mainstay of therapy is regular use of emollients and topical corticosteroids, but the long-term use of topical corticosteroids is limited by cutaneous atrophy, striae, and telangiectasia and the potential for systemic effects such as reduced bone density and suppression of the hypothalamicpituitary-adrenal axis. For more severe cases, phototherapy and immunosuppressants such as systemic steroids and calcineurin inhibitors have been used.1 Despite these therapies, many patients have refractory disease and thus, there is considerable interest in complementary medicines.

One such medicine of interest is Momordica charantia, which is a fruit that belongs to the Cucurbitaceae family. It is also known as bitter melon, bitter gourd, and balsam pear and grows in tropical areas of the Amazon, East Africa, Asia, India, South America, and the Caribbean. ${ }^{2}$ It is used in systems of traditional medicine and is commonly prepared as cooked fruit, tea, powdered fruit extract, or juice or blended extract. There is some evidence to support its medicinal use in diabetes, dyslipidemia, and certain infections and cancer, 2,3 but less is known about its medicinal value in atopic dermatitis, for which it is commonly used in other countries. There are no published reports in the English language scientific literature that evaluate its use for this indication. Here we report its benefit in a pediatric patient with severe refractory atopic dermatitis. The reporting of the case was approved by the institutional review board at the University of Tennessee Health Science Center.

\section{Case Report}

A 6-year-old Caucasian female presented with four years of severe refractory atopic dermatitis (Figure 1). Medical history was significant for allergic rhinitis and multiple food allergies. Family history was significant for maternal atopic dermatitis. Previous therapies included moisturizers, oral antihistamines, high potency topical corticosteroids, and four courses of oral steroids in the last year.

On physical exam, she was afebrile and her weight and height were above the $75^{\text {th }}$ percentile for her age. She had large patches of lichenification, erythema, xerosis, fissures, and excoriations on the front and back of her arms and legs; her wrists, hands, and feet were particularly affected. Focal areas of skin atrophy and tightness limited the range of motion in both her knees and hands (the distal interphalangeal joints of her index fingers) by about 30 degrees. Her SCORAD (SCORing Atopic Dermatitis), which is a validated clinical index to score the extent and severity of atopic dermatitis, ${ }^{4}$ was 72.8 out of a maximum score of 103 , consistent with severe atopic dermatitis.

Her antinuclear antibody and anti-scleroderma-70 antibody were negative, her total IgE was markedly elevated at $1,797 \mathrm{IU} / \mathrm{mL}$ (normal $0-393$ ), and her peripheral eosinophil count was $700 / \mathrm{mm}^{3}$ (normal $0-300$ ). Nasal swab culture had heavy growth of methicillin-sensitive Staphylococcus aureus. Allergy skin tests were positive for multiple food and inhalant allergens.

The patient was treated with emollients, oral antihistamines, and specific allergen avoidance measures. She was started on topical mupirocin to the nares and twice weekly dilute bleach baths to reduce Staphylococcal colonization. We suggested a trial of pimecrolimus $1 \%$ cream, which is a calcineurininhibitor approved for atopic dermatitis. It works similarly to tacrolimus and cyclosporine by inhibiting T-cell activation to suppress the
Correspondence: D. Betty Lew, Children's Foundation Research Center at Le Bonheur Children's Hospital, University of Tennessee Health Science Center, 50 North Dunlap Street, Rm 401, Research Tower, Memphis, Tennessee 38103, USA. E-mail: dlew@uthsc.edu

Key words: atopic dermatitis, Momordica charantia, case report, complementary therapies.

Conflict of interests: the authors report no conflict of interests.

Acknowledgments: we are grateful to the patient and her family for their contribution. We thank Dr. Jason B. Spencer for his assistance with the literature search.

Received for publication: 18 January 2012.

Revision received: 6 April 2012.

Accepted for publication: 11 April 2012.

This work is licensed under a Creative Commons Attribution NonCommercial 3.0 License (CC BYNC 3.0).

(C) Copyright D. Park et al., 2012

Licensee PAGEPress, Italy

Alternative Medicine Studies 2012; 2:e7

doi:10.4081/ams.2012.e7

immune response. The mother had heard about the benefits of using bitter melon from a Vietnamese family so we opted to try the pimecrolimus $1 \%$ cream twice daily to lesions on the right side of the body and topical bitter melon three times daily to lesions on the left side of the body. The mother prepared the bitter melon at home by taking $3-4$ fresh bitter melons, boiling them until they were about the consistency of boiled squash, letting them cool, pureeing them in their entirety - including peel, pulp, and seeds - then putting them through a strainer. The result was a semitransparent, stew-like paste that she rubbed into the patient's skin. It was left on and seemed to be fairly well-absorbed by the skin. Any remaining extract, she kept refrigerated for use later that week. Compliance was very good, as evidenced by the used tubes of pimecrolimus and the four bitter melons that the patient reportedly went through each week. The patient's mother was careful to apply the drug and complementary therapies on the designated side of the body.

In one month, the patient returned significantly better, with equal improvement on the pimecrolimus treated side (total use was about 100 grams in one month) as compared to the bitter melon treated side (Figure 1). She now had full range of motion in both knees, better range of motion in her index fingers, and a markedly improved SCORAD of 44.6. In addition, the skin on the bitter melon treated side 

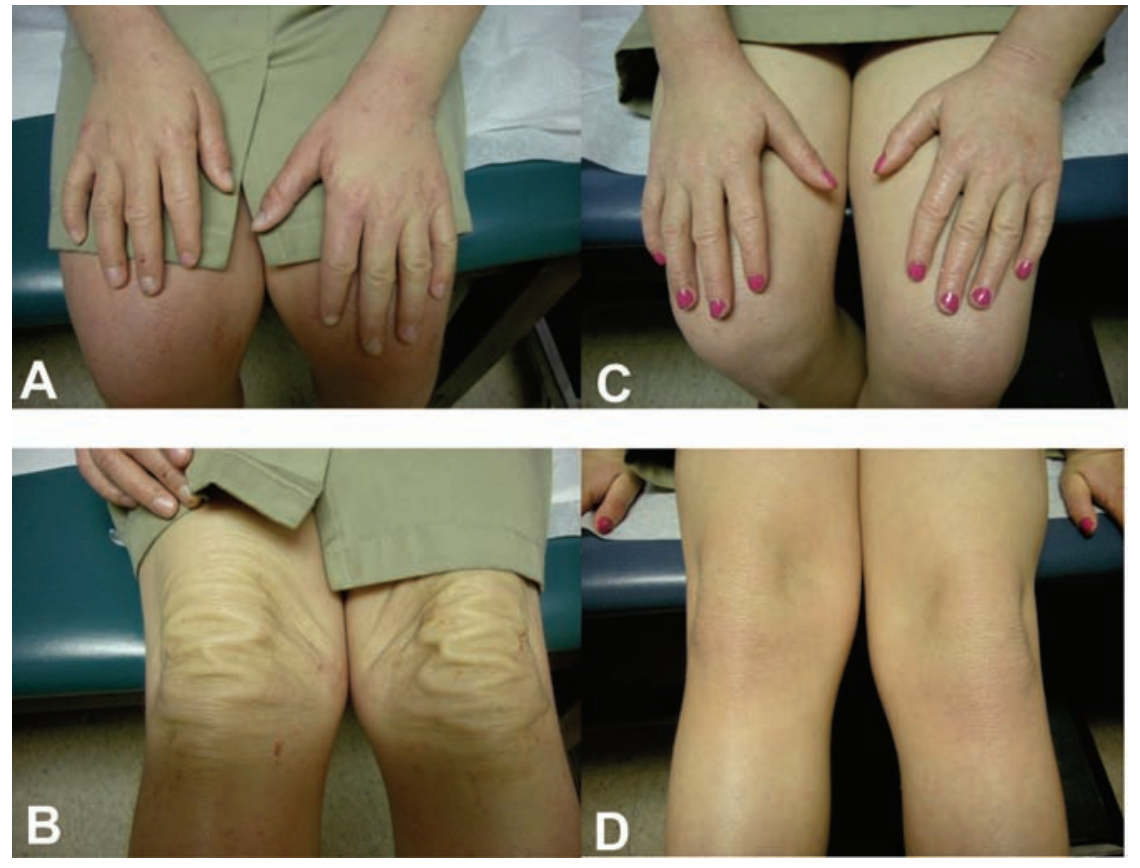

Figure 1. Benefits of pimecrolimus versus topical bitter melon in atopic dermatitis. Results of an open half-side comparison trial between pimecrolimus (1\% cream twice daily to right side of body) and topical bitter melon (extract 3 times daily to left side body) are shown. Images (A) and (B) are at baseline; (C) and (D) are one month after treatment. After treatment, there is marked improvement in erythema, edema, range of motion, scaling of the hands, and skin atrophy of the knees. In addition, the skin on the bitter melon treated side is softer than that on the pimecrolimus treated side.

was softer to the touch than on the pimecrolimus treated side. There were no reported adverse effects, other than a mild transient burning sensation that occurred with both the pimecrolimus and bitter melon application. There was also a pungent smell to the bitter melon that the patient noticed. Two years later, she continues to use the bitter melon extract intermittently, about 3-4 times a year for a few weeks at a time, with a good response and no adverse effects.

\section{Discussion}

Topical bitter melon may be a beneficial steroid- and pimecrolimus-sparing complementary medicine for severe atopic dermatitis in some patients, particularly those who do not respond to standard therapies or for whom the long-term use of such therapies are limited by adverse effects. Here our patient saw marked improvement, comparable to that seen with the potent immunosuppressant pimecrolimus, with one month of use.

In terms of side effects and toxicity, inges- tion of bitter melon is relatively safe as it is regularly consumed in the diet of many Asian countries. However, it has been known to cause hypoglycemia, interfere with pregnancy, and cause hemolytic anemia in persons with inherited deficiency of glucose-6-phosphate dehydrogenase.2,3,5 Ingestion of high concentrations of the extract may cause gastric ulceration and bleeding. ${ }^{6}$ Side effects with topical use are unknown, but may include a burning sensation and phytodermatitis.

There are a number of potential mechanisms by which bitter melon may exert its effects in atopic dermatitis. It may modulate anti-inflammatory cytokines, as studies show that the pulp upregulates interferon- $\gamma$ in mice, ${ }^{7}$ and transforming growth factor- $\beta$ and interleukin-10 in vitro. ${ }^{8}$ It has been shown to have anti-staphylococcal activity in vitro. ${ }^{9}$ It may also improve skin barrier function through effects on peroxisome proliferatoractivated receptor-delta, ${ }^{10}$ which is emerging as a player in epidermal barrier homeostasis. ${ }^{11}$ Further studies are needed to isolate the phytocomponents that mediate these effects and to determine what bearing they have on atopic dermatitis.

\section{References}

1. Hanifin JM, Cooper KD, Ho VC, et al. Guidelines of care for atopic dermatitis, developed in accordance with the American Academy of Dermatology (AAD)/American Academy of Dermatology Association Administrative Regulations for Evidence-Based Clinical Practice Guidelines. J Am Acad Dermatol 2004;50: 391-404.

2. Momordica charantia (bitter melon). Monograph, Altern Med Rev 2007:12:360-3.

3. Grover JK, Yadav SP. Pharmacological actions and potential uses of Momordica charantia: a review. J Ethnopharmacol 2004; 93:123-32.

4. Schmitt J, Langan S, Williams HC, European Dermato-Epidemiology Network. What are the best outcome measurements for atopic eczema? A systematic review. J Allergy Clin Immunol 2007;120: 1389-98.

5. Basch E, Gabardi S, Ulbricht C. Bitter melon (Momordica charantia): a review of efficacy and safety. Am J Health Syst Pharm 2003;60:356-9.

6. Nadkarni N, D'Cruz S, Sachdev A. Hematemesis due to bitter melon (Momordica charantia) extract-induced gastric ulcerations. Indian J Gastroenterol 2010;29:37-8.

7. Ike K, Uchida Y, Nakamura T, Imai S. Induction of interferon-gamma (IFNgamma) and $\mathrm{T}$ helper 1 (Th1) immune response by bitter gourd extract. J Vet Med Sci 2005;67:521-4.

8. Manabe M, Takenaka R, Nakasa T, Okinaka 0 . Induction of anti-inflammatory responses by dietary Momordica charantia L. (bitter gourd). Biosci Biotechnol Biochem 2003;67:2512-7.

9. Mwambete KD. The in vitro antimicrobial activity of fruit and leaf crude extracts of Momordica charantia: a Tanzania medicinal plant. Afr Health Sci 2009;9:34-9.

10. Sasa M, Inoue I, Shinoda Y, et al. Activating effect of momordin, extract of bitter melon (Momordica Charantia L.), on the promoter of human PPARdelta. J Atheroscler Thromb 2009;16:888-92.

11. Schmuth M, Jiang YJ, Dubrac S, et al. Thematic review series: skin lipids. Peroxisome proliferator-activated receptors and liver $\mathrm{X}$ receptors in epidermal biology. J Lipid Res 2008;49:499-509. 